\section{$\underset{\substack{\text { hommes } \\ \text { \& migrations }}}{ }$}

\section{Hommes \& migrations}

Revue française de référence sur les dynamiques

migratoires

$1313 \mid 2016$

1983, le tournant médiatique

\title{
Brève histoire du racisme en images
}

\section{Magdalena Ruiz-Marmolejo}

\section{(2) OpenEdition \\ Journals}

\section{Édition électronique}

URL : http://journals.openedition.org/hommesmigrations/3584

DOI : 10.4000/hommesmigrations.3584

ISSN : 2262-3353

\section{Éditeur}

Musée national de l'histoire de l'immigration

\section{Édition imprimée}

Date de publication : 1 janvier 2016

Pagination : 136-139

ISBN : 978-2-919040-34-6

ISSN : $1142-852 X$

\section{Référence électronique}

Magdalena Ruiz-Marmolejo, "Brève histoire du racisme en images », Hommes \& migrations [En ligne], 1313 | 2016, mis en ligne le 17 juin 2016, consulté le 15 septembre 2020. URL : http:// journals.openedition.org/hommesmigrations/3584 


\title{
COLLECTIONS
}

\section{BRÈVE HISTOIRE DU RACISME EN IMAGES}

\begin{abstract}
MAGDALENA RUIZ-MARMOLEJO, conservatrice du patrimoine au service des collections du Musée national de l'histoire de l'immigration.
\end{abstract}

A u Musée national de l'histoire de l'immigration, trois types des collections dialoguent et présentent divers aspects de l'immigration en faisant résonner le témoignage ethnologique, la trace historique et la création artistique contemporaine. Parmi ces collections, trois acquisitions relevant de registres très différents permettent d'aborder les représentations d'étrangers qui peuvent êtres considérées comme racistes ou antisémites.

\section{L'Assiette au beurre ou la satire d'une immigration invisible}

De nos jours, être anglais à Paris n'est pas forcément visible, ni connoté. Mais, en 1903, il n'en va pas de même, comme le montre cette Une de la revue satirique L'Assiette au beurre.

L'Anglais représenté en buste se détache sur un fond orange vif. S'il porte un costume de ville, il semble pourtant négligé, car son bonnet d'intérieur glisse de ses cheveux filasse. Cet Anglais a les yeux clairs perdus dans le vague, les oreilles décollées et un nez extrêmement rouge contrastant avec sa longue moustache noire. Le dessin de Sancha illustre ici l'anglophobie ambiante puisqu'il le rattache sans concession à la catégorie des ivrognes. Les traits de l'homme sont exacerbés et stigmatisés. L'Anglais n'incarne pas les valeurs de décence et la sobriété prônées par la société française.

En ce début du XXe siècle, Paris est déjà un véritable carrefour artistique. Les artistes qui collaborent à L'Assiette au beurre viennent de tous les horizons, tels que l'Hollandais Van Dongen, l'Italien Cappiello, le Suisse Vallotton, etc. Sancha est, quant à lui, espagnol. D’abord formé à l'École des Beaux-Arts de Malaga, il est ensuite l'élève de Juan Gris, peintre cubiste'. Dans ce contexte, la revue a consacré près de 1300 dessins dédiés aux diverses figures de l'étranger ${ }^{2}$. Avec ce titre de Une "Les Anglais chez nous ", la revue satirique exprime une appréhension présente dans la société française de l'époque. La puissance de l'Empire britannique est à la fois redoutée et tournée en dérision à la moindre occasion.

Héritée de la Révolution, généralement publiée dans la presse ${ }^{3}$, la caricature témoigne avec humour d'un point d'actualité ou des débats qui agitent la société française et tiennent compte de la circulation des idées. Ainsi, L’Assiette au beurre critique, entre autres, l’armée, la police, la justice, les députés, le clergé, le travailleur... Le discours 


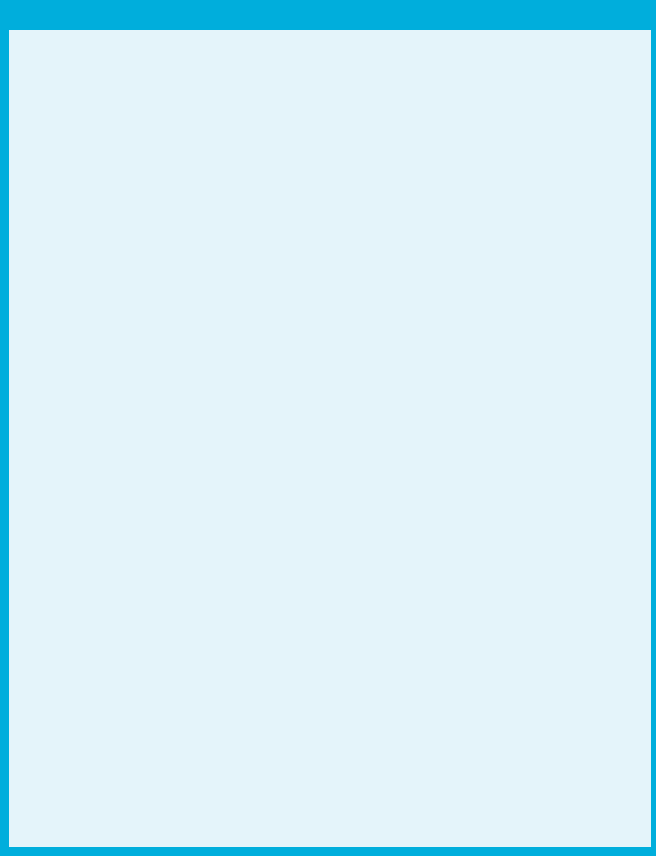

Francisco Sancha y Lengo, dit Sancha (1874-1936)

"Les Anglais chez nous ", L'Assiette au beurre, n92,

03 janvier 1903, impression par photogravure $31,3 \times 24,2 \mathrm{~cm}$

(c) Musée national de L'Histoire de l'immigration.

est autant visuel qu'écrit, car la caricature utilise la même grammaire que le calembour graphique. Elle allégorise en employant la métaphore ${ }^{4}$. Claude Lévi-Strauss explique dans La Voie des masques que la caricature est une forme artistique anti-canonique, mais qui reste liée aux valeurs de la société qu'elle dénonce.

C'est pour cette raison que la caricature est considérée comme une source d'informations privilégiée sur l'opinion publique, sur les stéréotypes, mais aussi sur les imaginaires politiques et sociaux. Christian Delporte la qualifie d'ailleurs de "forme d'expression visuelle éminemment représentative de la civilisation industrielle et de la culture de masse ${ }^{5} »$. Les historiens ont donc été les premiers à l'étudier ; ce qui explique, par ailleurs, que les recherches sur les caricatures adoptent une démarche chronologique et, depuis les années 1970, une approche plus interdisciplinaire.

\section{L'affiche du savon Dirtoff ou la représentation du Noir dans la publicité}

Cette affiche présente un jeune homme de couleur en train de se laver les mains au dessus d'un lavabo. La boîte de savon utilisée est mise en valeur au premier plan. Le dessin est accompagné d'une inscription, « pour mécaniciens automobilistes et ménagères ", "nettoie tout ", qui fait écho à celle en partie supérieure "Le savon Dirtoff me blanchit!". Les traits grossiers, la bouche énorme, le sourire forcé et le pantalon rayé accentuent l'effet clownesque du personnage. La scène semble à la fois burlesque et presque effrayante car le savon décolore vraiment les mains de l'homme noir. Comme dans d'autres publicités, telles que celles pour le chocolat Banania ou Menier - pour ne citer que les plus célèbres -, les personnes de couleur sont très présentes dans les affiches des années 1920-19306. Les productions des colonies y sont mises en valeur et montrées comme issues de terres lointaines, exotiques et peuplées de sauvages.

En réalité, par ces images racisées, la publicité diffuse massivement le discours impérialiste et banalise des clichés idéologiques. Ici, la noirceur de la peau est considérée comme une saleté dont il faut se débarrasser : I'homme noir singe l'homme blanc et, intrinsèquement, le considère comme un modèle dont il lui faut se rapprocher. Cette hiérarchie des hommes par la couleur de peau n'est pas sans rappeler celle des tableaux de castes où les métissages de la Nouvelle-Espagne sont décrits et classés par degrés. Ce racisme de couleur a également une origine religieuse. En effet, suite à la conquête des Amériques, la bulle papale Sublimis Deus de 1537 accorde l'humanité aux Amérindiens. De ce fait, la traite des Noirs 


\section{COLLECTIONS}

vers les Amériques est généralisée et amplifiée. D'ailleurs, cet imaginaire raciste se trouve renforcé par les diverses expositions coloniales qui ont rythmé l'entre-deux-guerres : à Marseille
Les signes extérieurs de la personne sont les premières cibles - couleur de peau, accent, religion, tenue vestimentaire, habitude alimentaire -, et cela malgré l'adoption de la loi sur la liberté de la presse du 29 juillet 1881. Le discours raciste contre les colonisés est donc largement véhiculé par les publicités qui à la fois reflètent l'imaginaire sociale et contribuent à le renforcer. Finalement, il faudra attendre la loi du $1^{\text {er }}$ juillet 1972 pour que les injures raciste ou antisémites soient punies en France.

\section{Les marques \\ de l'antisémitisme captées par la photographie contemporaine}

En travaillant à l'agence Magnum dans les années 1980, Patrick Zachmann adopte le sens de la composition narrative?. À la même époque, il s'interroge sur son propre métissage culturel et mène une enquête photographique sur les rituels et les appartenances aux communautés ${ }^{8}$. Sur cette photographie, il capte en image une tombe juive dégradée par la croix gammée, dont il enregistre ainsi l'acte, à la fois volontaire et violent, porteur d'un message politique. L'antisémitisme avait connu une forte virulence au XIXe siècle, notamment avec les pogroms russes et l'affaire Dreyfus, et avait abouti au nazisme et à la Shoah au XXe siècle. Les jeunes années de Patrick Zachmann sont troublées par les groupuscules fascistes et les conflits internationaux. En France, les agressions et manifestations antisémites se multiplient... Le point d'orgue a lieu en 1980, lors de l'attentat de la synagogue de la rue Copernic à Paris. Le cimetière est en 1922, à Strasbourg en 1924 et à Paris en 1931 au Palais de la Porte Dorée. L'étranger, qu'il soit immigré ou non, est donc victime de racisme.
Anonyme, Le savon Dirtoff nettoie tout, vers 1920 affiche lithographie entoilée, $100 \times 70 \mathrm{~cm}$ (c) Musée national de L'Histoire de L'immigration. 
Patrick ZACHMANN, Inscriptions antisémites..., 1981, Tirage sur papier argentique,

50 X $40 \mathrm{~cm}$ @ Musée national de L'HISTOIRE DE L'IMMIGRATION, 2006.45.1

considéré comme un lieu sacré. Lui porter atteinte est identifié comme un acte de profanation.

Il est donc possible de le rapprocher des actes iconoclastes perpétrés sur des statues ou tableaux dont ils partagent le même but : anéantir le pouvoir de ce qui est représenté. Qu'ils soient liés à du fanatisme religieux ou politique, les profanations et actes d'iconoclasme sont toujours d'actualité et leur dimension s'est internationalisée. C'est donc à chacun de s'interroger sur la possibilité de conserver ce qui fait sa propre identité. Force est de constater que l'histoire du racisme et de l'antisémitisme fait partie intégrante de l'histoire de France. Mais on ne nait pas raciste, on le devient. La diffusion des connaissances sur l'histoire du racisme et de l'antisémitisme et l'éducation apparaissent comme des approches essentielles dans la lutte contre ces formes de domination sur d'autres personnes. Par ailleurs, si la diversité culturelle s'avère constituante de l'humanité, le fait d'affirmer que toutes les cultures sont de même valeur est récent. Ce principe désigné par Claude Lévi-Strauss sous le terme de " relativisme culturel » doit permettre à chaque culture de se développer, de sorte que " chacune soit une contribution à la plus grande générosité des autres ${ }^{9}$ ". C'est à ces missions que le Musée national de l'histoire de l'immigration s'attelle par la présentation et l'analyse de ses collections. I 